\title{
Fission fragment formation and fission yields in the model of octupole neutron-proton oscillations
}

\author{
S.Yavshits, ${ }^{\text {a }}$ \\ V.G.Khlopin Radium Institute, St.-Petersburg, Russia
}

\begin{abstract}
The fission fragment formation is considered as a result of neck instability in the process of octupole oscillations of neutrons and protons near the scission point. To describe such a phenomenon the potential surface of fissionning nucleus with neck radius about $1 \mathrm{fm}$ was calculated with shell correction approach. The new version of smooth liquid drop part of deformation energy is proposed. The liquid drop part is formulated in a double folding model with $n-n, p-p$, and n-p Yukawa interaction potential. Fission fragment mass and charge distributions correspond approximately to isoscalar and isovector modes of vibrations and are defined by wave functions of oscillations. The preliminary calculation results have shown a rather good description of main integral fission yield observables.
\end{abstract}

\section{Introduction}

Fission fragment formation is still one of amazing and poorly investigated issues of fission physics. Division of fissioning nucleus into two massive fragments is tightly bound with instabilities developing in nuclear matter relative to possible ruptures and one can expect that probability of such a rupture is most probable near the scission point where the neck radius is close to nucleon radius. Distributions of fragments on mass and charge degrees of freedom will be defined by oscillations of neutron and proton components of nuclear density along corresponding collective coordinates (parameters of shape deformations) governed mainly by potential energy of oscillations near the scission point.

\section{Model}

One of the important points in the study of nuclear configurations near the scission point is the choice of shape parameterization. We use shape parameterization proposed in [1] for axially symmetrical configurations. Here nuclear shape is defined in the orthogonal coordinate system where basic family of coordinate surfaces is deformed ovals of Cassini allowing to describe both oblate and prolate shapes including strongly prolate ones right up to division of nucleus on two fragments. For our goals we will use three main parameters of deformations that are $\{\alpha\}=\left(\alpha, \alpha_{3}, \alpha_{4}\right)$ where $\alpha$ is the lemniscate parameter, $\alpha_{3}$ defines octupole mirror asymmetry of nuclear shape and $\alpha_{4}$ is the parameter of hexadecapole deformation. At small values of the lemniscate parameter the shape of nucleus looks like ovaloids while values $\alpha>0.9$ corresponds to configurations with developed neck.

\footnotetext{
${ }^{a}$ e-mail: yav@mail.rcom.ru
}

Governing value in our model is the dependence of the potential energy on $\alpha_{3}$ near the scission point. We use the Strutinsky prescription for energy calculations where the potential energy is a sum of smooth liquid drop part and shell and pair corrections [2]. With taking into account pairing the potential energy is:

$$
V_{p o t}\{\alpha\}=E_{l d}\{\alpha\}+\delta V\{\alpha\}+E_{\text {pair }}\{\alpha\}
$$

where $E_{l d}$ is a liquid drop energy, $\delta V$ is shell correction and $E_{\text {pair }}$ is the pair energy.

For calculations of charge distributions the new version of liquid drop model has been applied. The model is based on double folding approach of nuclear density with Yukawa potential. Similar approach has been proposed in the early works of Krappe-Nix [3] and was developed further by the group of Pomorski (see [4] and references therein). In this approach

$$
E_{l d}=\frac{1}{2 \rho_{0}^{2}} \iint \rho\left(\mathbf{r}_{1}\right) \rho\left(\mathbf{r}_{2}\right) \vartheta\left(\left|\mathbf{r}_{1}-\mathbf{r}_{2}\right|\right)
$$

where $\rho$ is the nucleon density in the point $\mathbf{r}$ and $\vartheta$ is the Yukawa' potential for nucleon-nucleon interactions depending from the mutual distance between two nucleons:

$$
\vartheta\left(\left|\mathbf{r}_{1}-\mathbf{r}_{2}\right|\right)=-\frac{\vartheta_{0}}{4 \pi a^{3}} \frac{e^{-\left|\mathbf{r}_{1}-\mathbf{r}_{2}\right| / a}}{\left|\mathbf{r}_{1}-\mathbf{r}_{2}\right| / a}
$$

If nucleons are indistinguishable, eq. (3) reduces to the standard liquid drop model. Taking into account that $\rho=$ $\rho_{n}+\rho_{p}$, (3) may be presented as

$$
\begin{aligned}
& E_{l d}=E_{l d}^{n n}+E_{l d}^{p p}+\frac{1}{2} E_{l d}^{p n}, \quad E_{l d}^{q q^{\prime}}=\frac{1}{2 \rho_{0}^{2}} \mathbf{r}_{2} \rho_{q^{\prime}}\left(\mathbf{r}_{2}\right) \vartheta\left(\left|\mathbf{r}_{1}-\mathbf{r}_{2}\right|\right), \\
& q \equiv(p, n)
\end{aligned}
$$

This is an Open Access article distributed under the terms of the Creative Commons Attribution-Noncommercial License, which permits unrestricted use, distribution, and reproduction in any noncommercial medium, provided the original work is properly cited. 
that is as a sum of macroscopic energies for neutron component of density, proton component and their interaction. Using Gauss-Ostrogradsky' theorem and suggesting axial symmetry of nucleus the integral in (4) (6-multiple in the common case) may be reduced to 3-multiple one [3]

$$
\begin{aligned}
& E_{l d}^{q q^{\prime}} \equiv E_{l d}\left(\{\alpha\}_{q},\{\alpha\}_{q^{\prime}}\right)=-\frac{\vartheta_{0}}{2 a} \int d z \int d z^{\prime} \int_{0}^{2 \pi} d \phi R_{q}(z) \times \\
& {\left[R_{q}(z)-R_{q^{\prime}}\left(z^{\prime}\right) \cos \phi-R_{q}^{\prime}(z)\left(z-z^{\prime}\right)\right] \times R_{q^{\prime}}\left(z^{\prime}\right) \times} \\
& {\left[R_{q^{\prime}}\left(z^{\prime}\right)-R_{q}(z) \cos \phi-R_{q}^{\prime}(z)\left(z-z^{\prime}\right)\right] \frac{d+(2+d) e^{-d}-2}{d^{4}},} \\
& d=\frac{1}{a}\left[R_{q}^{2}(z)+R_{q^{\prime}}^{2}\left(z^{\prime}\right)-2 R_{q}(z) R_{q^{\prime}}\left(z^{\prime}\right) \cos \phi+\left(z-z^{\prime}\right)^{2}\right]^{1 / 2},
\end{aligned}
$$

where $R_{q}(z)$ is the surface equation for neutron (proton) component in the cylindrical coordinates.

Finally the potential energy can be written as

$$
\begin{aligned}
& V_{T}\left(\{\alpha\}_{n},\{\alpha\}_{p}\right)=E_{l d}^{n n}\left(\{\alpha\}_{n}\right)+E_{l d}^{p p}\left(\{\alpha\}_{p}\right)+E_{l d}^{n p}\left(\{\alpha\}_{n},\{\alpha\}_{p}\right)+ \\
& f(T)\left[\delta E_{n}\left(\{\alpha\}_{n}\right)+\delta E_{p}\left(\{\alpha\}_{p}\right)\right],
\end{aligned}
$$

where $\delta E=\delta V+E_{\text {pair }}$ and function $f(T)$ reflects dumping of nuclear structure effects with nuclear temperature increase.

We used Woods-Saxon form of function $f(T), f(T)=$ $1 /\left(1+\exp \left(T-T_{c r}\right) / b\right), T_{c r}, \mathrm{~b}$ are model parameters.

\section{Results and discussion}

Results of potential surface calculations vs. asymmetry parameters of neutrons and protons for $\alpha=0.99$ and with minimization on the hexadecapole parameter $\alpha_{4}$ for cold fission of ${ }^{236} \mathrm{U}$ are presented in Figs. 1a,b. Results obtained show that there is a structure in the potential surface along line of equal asymmetry parameters $\alpha_{3}^{n}=\alpha_{3}^{p}$ and narrow minimum along lines $\alpha_{3}^{n}=-\alpha_{3}^{p}+$ const. It gives us possibility to introduce in the natural way new coordinate system $(x, y)$ by rotation of axes on angle equal to $45^{\circ}$ (see Fig.1b), $x=\frac{\sqrt{2}}{2}\left(\alpha_{3}^{n}+\alpha_{3}^{p}\right), \quad y=\frac{\sqrt{2}}{2}\left(\alpha_{3}^{n}-\alpha_{3}^{p}\right)$ or, introducing average asymmetry $\alpha_{3}=\frac{\alpha_{3}^{n}+\alpha_{3}^{p}}{2}$ and difference $\Delta \alpha_{3}=\frac{\alpha_{3}^{n}-\alpha_{3}^{p}}{2}, x=\sqrt{2} \alpha_{1}, \quad y=\sqrt{2} \Delta \alpha_{3}$. Then $\alpha_{3}^{n}=\alpha_{3}+\Delta \alpha_{3}, \quad \alpha_{3}^{p}=\alpha_{3}-\Delta \alpha_{3}$, i.e. at $\Delta \alpha_{3}=0$ neutrons and protons move in phase with equal asymmetry parameters and at $\Delta \alpha_{3} \neq 0$ they are in antiphase relatively to average value $\alpha_{3}$ corresponding to the minima of the potential energy.

Parameters of asymmetry $\alpha_{3}^{q}$ are connected with nucleon numbers $N_{q}$ by the following integral

$$
N_{q}^{f}=2 \pi \frac{N_{q}^{F}}{V} \int_{z_{\min }}^{z_{1}} R_{q}^{2}(z) d z,
$$

where $N_{q}^{f}$ one of fragments, $N_{q}^{F}$ is the number of neutrons (protons) for fissioning nucleus, $V$ is equal to its volume, $z_{\min }$ is the value of coordinate $z$ in the point with minimal value of neck radius and $z_{1}$ is the value of $z$ on the
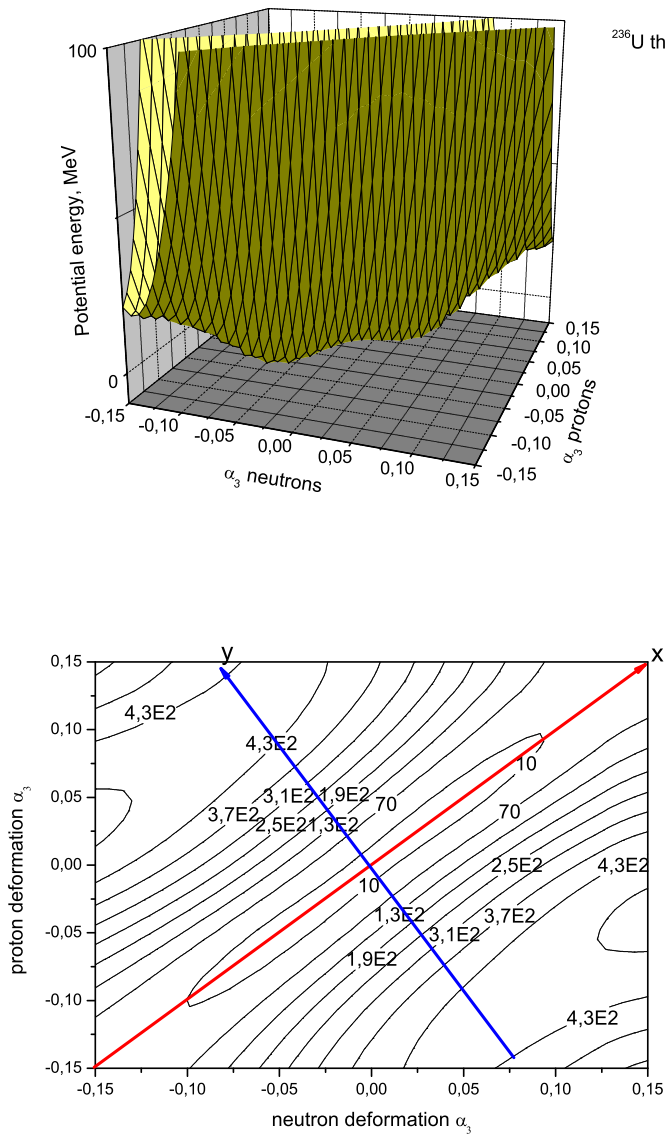

Fig. 1. a,b. Surface and map of potential energy vs. asymmetry parameters of neutrons and protons.

nuclear edge. For our shape parameterization such a dependence is almost linear, $\alpha_{3}^{q} \approx c N_{q}^{f}$. Then condition $y=$ const will correspond to condition $N_{f}-Z_{f}=$ const, that is to constant isospin and variable mass of fragment, and condition $x=$ const corresponds to $N_{f}+Z_{f}=A_{f}=$ const, that is constant mass and variable charge and neutron numbers of fragment. So, the significant physical conclusion may be done: fragment mass distributions are formed along lines of equal isospin and are a result of isoscalar mode of neutronproton oscillations while isobaric distributions (charge distribution with constant fragment mass) are formed along lines of equal mass and are a result isovector mode of oscillations.

It is necessary in the common case to solve two-dimensional wave equation in the plane $(x, y)$ in order to find out mass and charge distributions,

$$
\begin{aligned}
& \left\{-\frac{\hbar^{2}}{2 \sqrt{B_{x}}} \frac{\partial}{\partial x} \frac{1}{\sqrt{B_{x}}} \frac{\partial}{\partial x}-\frac{\hbar^{2}}{2 \sqrt{B_{y}}} \frac{\partial}{\partial y} \frac{1}{\sqrt{B_{y}}} \frac{\partial}{\partial y}+V(x, y)\right\} \psi_{v}(x, y) \\
& =E_{v} \psi(x, y)
\end{aligned}
$$


where $B_{x, y}$ are mass parameters of oscillations, $V(x, y)$ is the potential energy, $E_{v}, \psi_{v}$ are energies and wave functions.

However, the structure of potential surface (Fig. 1) allows to consider oscillations as independent ones with sufficient accuracy and solve a system of two unbound equations.

Mass and charge spectra for low-energy fission will be defined by wave function of corresponding oscillations

$$
Y(q) \propto \sum_{v}\left|\Psi_{v}(q)\right|^{2}\left(e^{E v_{1}} T-1\right)^{-1} d q, \mathrm{q} \equiv(\mathrm{x}, \mathrm{y})
$$

The model developed has been verified in the test calculation and comparison with experimental data for thermal fission of uranium and plutonium. Results of such a comparison are displayed in Figs. 2-7.

As can be seen from the figures, the results of the calculations are in a good agreement with basic integral data on mass and charge distributions of fragments. At the same time, the calculations did not reproduce the structural effects observed in the average values and widths of isobaric charge distributions (Fig. 6,7), although they close to averages. The average value of the charge in the isobaric chain shown in Fig. 4a in the form of a standard deviation of the charge made in the assumption of constant charge density $Z_{U C D}=\frac{Z}{A} A_{f}, \quad Z, A, A_{f}$ are charge and mass of fissile nucleus and fragment, respectively.

Reason for such a discrepancy can be related to the incomplete account of the effects of nuclear structure, especially in the effective mass in the equation (7). The impact of such effects as well as temperature effects will be considered at the next stages of this work.

The work was partly done under ISTC N3751 project.

\section{References}

1. V.V.Pashkevich, Nucl.Phys. A, 169, (1969) 275

2. V.M.Strutinsky, Nucl.Phys. A, 122, (1968) 1

3. H.J.Krappe and R.Nix, Proc. Third IAEA Symposium on Physics and Chemistry of Fission (IAEA Rochester 1973) 159

4. A.Dobrowolski, K.Pomorski and J.Bartel, Phys. Scr. textbf T125 (2006) 188

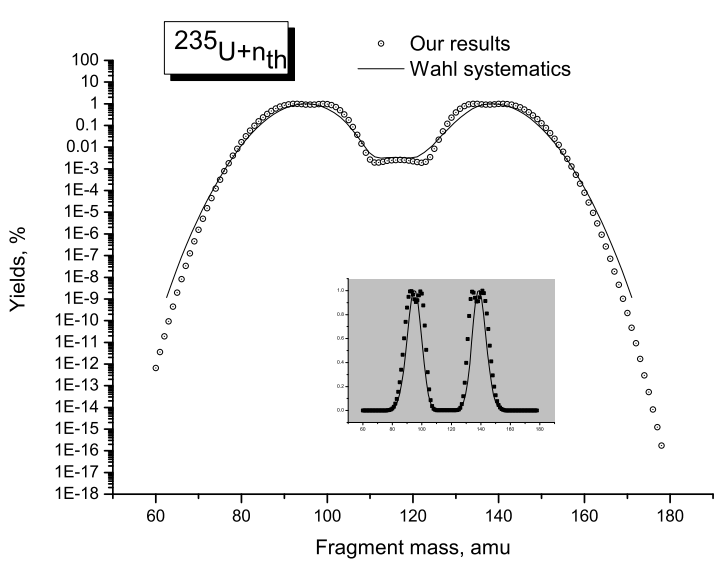

Fig. 2. Mass distributions of fission fragments for uranium. Results in line scale are presented in insertions.

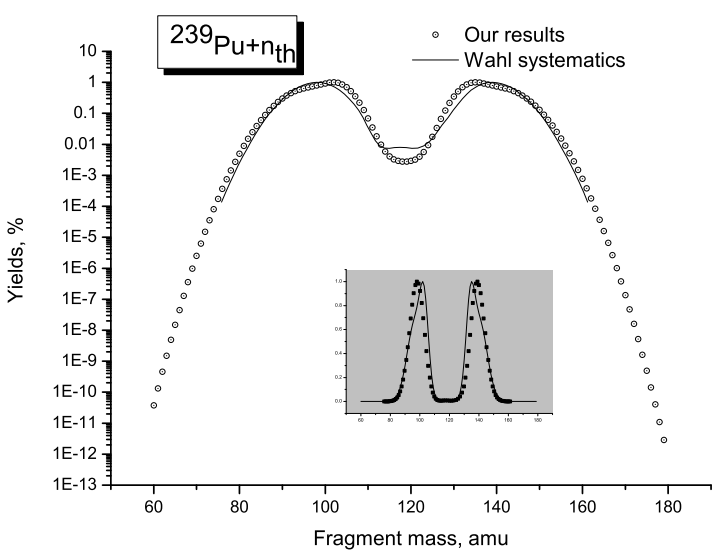

Fig. 3. Mass distributions of fission fragments for plutonium. Results in line scale are presented in insertions.

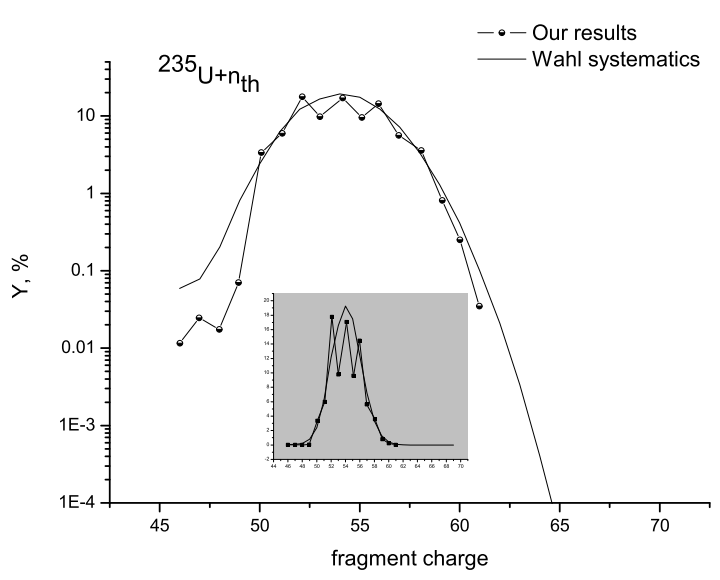

Fig. 4. The same as in the Fig. 2 but for charge distribution. 


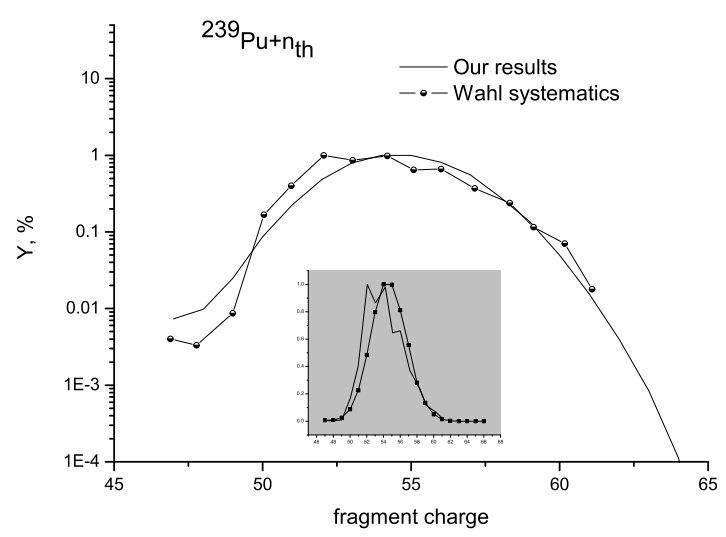

Fig. 5. The same as in the Fig. 3 but for charge distribution.

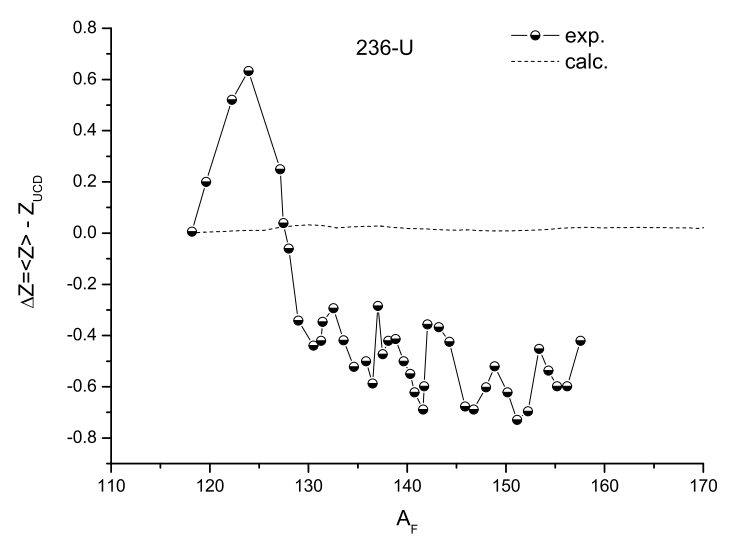

Fig. 6. Average charge values of isobaric charge distributions.

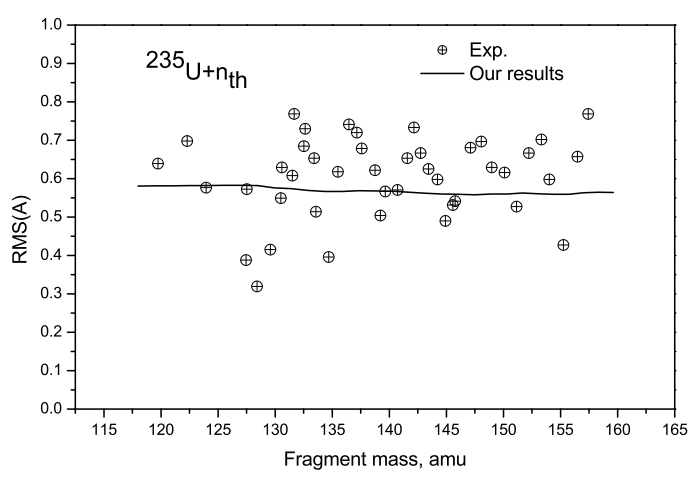

Fig. 7. Mean widths of isobaric charge distributions. 